\title{
Admittance spectroscopy of polymer-nanoparticle nonvolatile memory devices
}

\author{
D. T. Simon and M. S. Griffo \\ Physics Department, University of California, Santa Cruz, 1156 High Street, Santa Cruz, California 95064
}

R. A. DiPietro and S. A. Swanson

IBM Research Divison, Almaden Research Center, 650 Harry Road, San Jose, California 95120

S. A. Carter ${ }^{\mathrm{a}}$

Physics Department, University of California, Santa Cruz, 1156 High Street, Santa Cruz, California 95064

(Received 28 June 2006; accepted 1 August 2006; published online 28 September 2006)

\begin{abstract}
Nonvolatile resistive memory consisting of gold nanoparticles embedded in the conducting polymer poly(4- $n$-hexylphenyldiphenylamine) examined using admittance spectroscopy. The frequency dependence of the devices indicates space-charge-limited transport in the high-conductivity "on" state, as well as evidence for similar transport in the lower-conductivity "off" state. Furthermore, the larger dc capacitance of the on state indicates that a greater amount of filling of the midgap nanoparticle trap levels increases the overall device conductivity, leading to the memory effect.
\end{abstract}

(C) 2006 American Institute of Physics. [DOI: 10.1063/1.2357560]

The demand for faster, cheaper, denser memory technology and the accelerating pace of the semiconductor industry have driven an expansion of information storage research. In addition to novel approaches to scaling of existing technology, ${ }^{1-3}$ candidates for entirely different storage media are being investigated. In particular, several solid-state nonvolatile low power consumption structures are being actively pursued, e.g., magnetic random access memory, ${ }^{4}$ ferroelectric random access memory, ${ }^{5}$ phase-change (ovonic unified memory), ${ }^{6}$ and polymeric/organic. ${ }^{7-11}$ Many of these structures leverage a two-terminal-per-bit cross-point architecture to simplify the fabrication process and reduce the effective footprint of each bit.

Nonvolatile memory based on metallic nanoparticles embedded in a polymer host has been suggested ${ }^{8,9}$ as one of these new cross-point memory structures. In this system, trap levels situated within the band gap of the polymer are introduced by the nanoparticles. It has been theorized that the memory effect is due to the charge state of these traps but the exact relationship between this charge state and the device conductivity remains unclear. As these midgap traps should affect the frequency response of the device, we have applied the techniques of admittance spectroscopy to elucidate the correlation between device conductivity and the introduction of metallic nanoparticle trap states.

In this letter, we focus on devices consisting of a blend of triphenylphosphine-protected $\mathrm{Au}$ nanoparticles $(\sim 4 \mathrm{~nm}$ diameter) (AuNP) and the cross-linkable conducting polymer poly(4- $n$-hexylphenyldiphenylamine) ( $x$ HTPA) sandwiched between $\mathrm{Al}$ electrodes (as reported by Bozano et al. ${ }^{12}$ ). Using a shadow mask, a $10 \mathrm{~nm}$ seed layer of $\mathrm{Cr}$, followed by $30 \mathrm{~nm} \mathrm{Al}$ electrode was thermally evaporated onto a glass substrate at about $10^{-6}$ torr. The substrates were then removed from vacuum, and a $1.74 \mathrm{wt} \% x$ HTPA:xylenes solution loaded with $2.5 \mathrm{wt} \%$ AuNP: $x$ HTPA was spin cast at $500 \mathrm{rpm}$ for $10 \mathrm{~s}$, followed by $800 \mathrm{rpm}$ for $30 \mathrm{~s}$, resulting in a $125 \mathrm{~nm}$ thick active layer. This $x$ HTPA:AuNP layer was

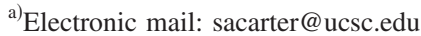

then annealed at $150{ }^{\circ} \mathrm{C}$ for $1 \mathrm{~h}$ to remove the solvent and cross-link the polymer. The devices were then returned to vacuum and a top $30 \mathrm{~nm} \mathrm{Al}$ electrode was evaporated. All fabrication and characterization was carried out under nitrogen.

The measurements were performed using a WaveTek 650 variable phase synthesizer to source a voltage across the device and a load resistor in series. When performing the dc current versus voltage $(I-V)$ sweeps, a Keithley 2010 multimeter measured the voltage drop across the load resistor to get the dc current. During the frequency sweeps, a Stanford Research Systems SR830 lock-in amplifier measured the voltage drop and phase shift across the load resistor. Dividing the ac current measured across the load resistor by the input voltage yields the complex admittance, $Y=I / V=G(\omega)$ $+i \omega C(\omega)$, the real and imaginary parts of which give the conductance $G(\omega)$ and capacitance $C(\omega)$. The input voltage for all frequency sweeps consisted of $50 \mathrm{mV} \mathrm{rms} \mathrm{ac} \mathrm{signal}$ with a $1 \mathrm{~V}$ dc bias, chosen to be at or near the expected "read" voltage. Low temperature measurements were conducted in an Oxford Instruments MagLab System 2000 cryostat.

The results of a typical room temperature dc measurement are shown in Fig. 1. The device exhibits three distinct regions of current-voltage relationship: (a) below some threshold voltage, $V_{\text {th }}$, at which the device switches conductance states, (b) a negative differential resistance (NDR) region, and (c) the region beyond NDR. Using a read voltage $<V_{\text {th }}$, at least two stable states are observed, which will be referred to in this letter as "on"-high conductivity-and "off"-low conductivity. The device can be set from off to on by applying a voltage equal to, or slightly greater than, $V_{\text {th }}$, and set from on to off by applying of a voltage near the end of the NDR region. Past NDR, the current appears to continue on a trend extending from the off state, which indicates that the same mode of conduction might be at play in these two regions.

It should be noted that the on-off current ratio of less than 1 order of magnitude in this device structure is low in 


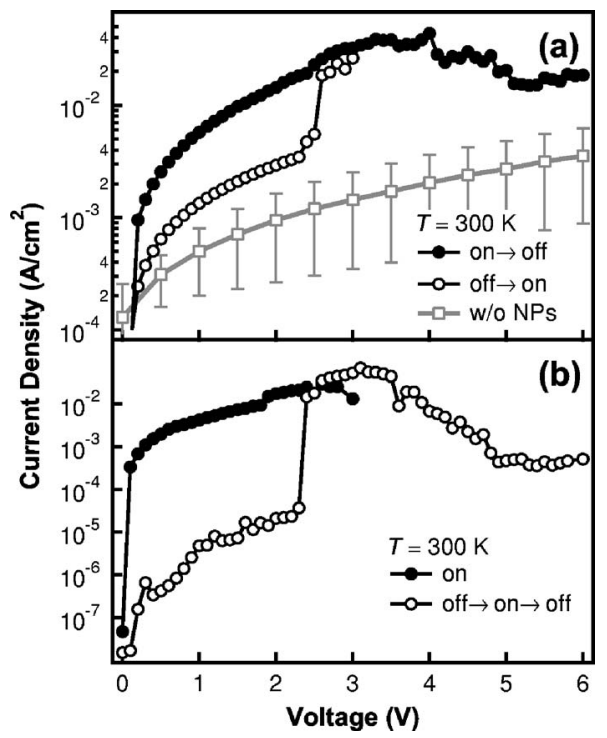

FIG. 1. Characteristic current vs voltage $(I-V)$ curves at room temperature exhibiting the bistable memory effect using (a) an Al/xHTPA:AuNP/Al structure and (b) a Au/MEH-PPV:AuNP/Au structure. In both cases the device is set on by sweeping past $V_{\text {th }}($ here, $\approx 2.5 \mathrm{~V}$ ) and off by continuing the sweep into the NDR region. The gray curve in (a) shows the average response (and standard errors) of a similar "clean" $x$ HTPA structure without NPs.

comparison to devices employing other polymer hosts. For example, poly(2-methoxy-5-(2'-ethylhexyloxy)-p-phenylene vinylene) (MEH-PPV) yielded on-off ratios ranging from 1 to 4 orders of magnitude (Fig. 1). This effect may be due to the cross-linkable nature of $x$ HTPA: the end groups intended for cross-linking may introduce sufficient trap sites to blur the distinction between the on and off states. Note that in Fig. 1(a), the averaged current of similarly prepared devices without NPs is below the off state of the NP-containing memory device, yet well above the off state of the MEHPPV:AuNP device. However, the $x$ HTPA device structure had the highest yield and resulted in the largest amount of collected data, therefore impedance measurements were performed on this structure.

The Cole-Cole plot of the impedance in the on and off states is shown in Fig. 2. The off state appears roughly semicircular, indicating that the device behaves somewhat as a

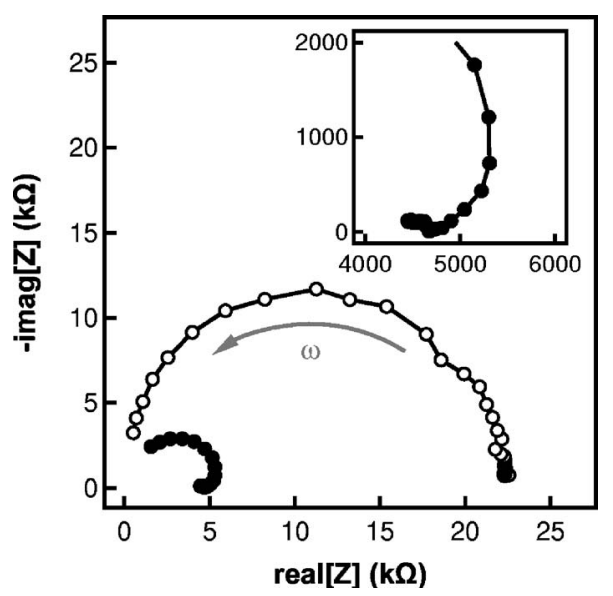

FIG. 2. Cole-Cole plot of imaginary vs real impedance for $(\bullet)$ on and $(\bigcirc)$ off states. The arrow indicates the direction of increasing frequency. The inset is a zoom showing the inductive bend (i.e., deviation from semicircle)

in the on state, which corresponds to the negative peak in Fig. 3.

03 Oct 2006 to 169.233 .62 .188 . Redistribution subject to
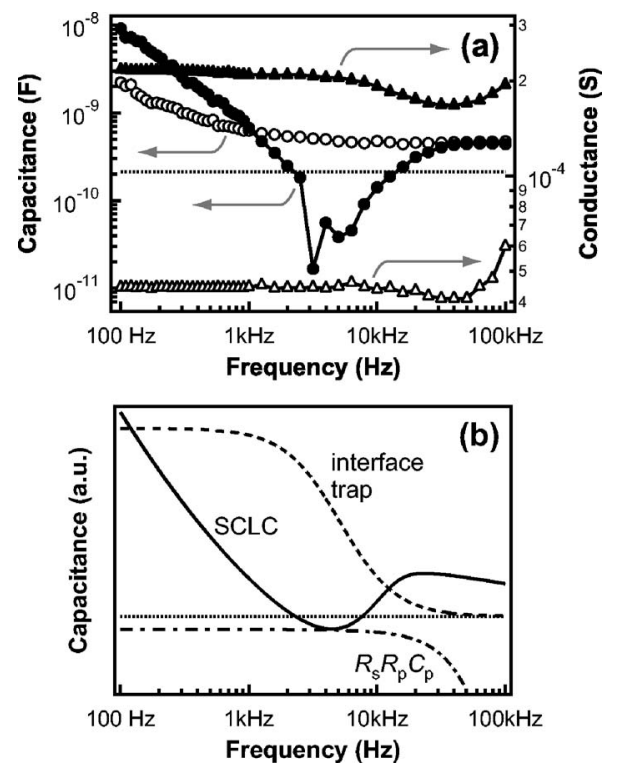

FIG. 3. (a) Capacitance and conductance as a function of frequency: (๑) on state capacitance, $(\mathbf{\Delta})$ on state conductance, $(\bigcirc)$ off state capacitance, and $(\triangle)$ off state conductance. (b) Capacitance vs frequency for three device models: interfacial trap layers (Ref. 13), space-charge limited current (Ref. $14)$, and a resistor in series with a parallel resistor-capacitor combination $\left(R_{s} R_{p} C_{p}\right)$. The horizontal dotted line in both plots shows the geometric capacitance, $C_{\text {geo }}=212 \mathrm{pF}$.

parallel $R C$ circuit. However, the on state shows a marked deviation from semicircular at low frequency, indicating that the $R C$ equivalent is not valid. The corresponding capacitance and conductance versus frequency are shown in Fig. 3. At low frequency, the capacitance in the on state is larger than in the off state. Given the presence of trap states, this greater ability to store charge indicates that these traps are more likely to be filled in the on state. The faster fall off of the on state capacitance with frequency is further evidence that a larger amount of charge-located at the interfaces, in the bulk, or both-cannot as easily keep up with the applied ac field. Finally, the presence of a negative (inductive) peak in the on state capacitance is indicative of space-chargelimited (SCL) behavior (cf. Fig. 3 and Refs. 14-16). The dips in the conductance of both the on and off states also qualitatively match SCL behavior. Following the analysis of Martens et al. ${ }^{14}$ the dc mobility can then be found using the time-of-flight argument, $\mu_{\mathrm{dc}}=L^{2} /\left(\tau_{t} V\right)$, for thickness $L$, transit time $\tau_{t}=1 / f_{\text {peak }}$, and dc bias $V$. Applying this equation to the on-state peak in Fig. 3(a), $\mu_{\mathrm{dc}}=7.2 \times 10^{-7} \mathrm{~cm}^{2} / \mathrm{V}$ s at $1 \mathrm{~V}$ bias.

At room temperature the characteristic sharp increase in conductivity at $V_{\text {th }}$ occurs consistently at $\sim 2.5 \mathrm{~V}$ and the NDR is observed over the range of $\sim 3-5 \mathrm{~V}$. On subjecting the device to low temperature $(110-250 \mathrm{~K})$ all device characteristics-location of $V_{\text {th }}$, sharpness of switch at $V_{\text {th }}$, and width of NDR - became sporadic or broadened. The $V_{\text {th }}$ occurred over $\sim 1.5-3 \mathrm{~V}$ (but was still mostly sharp) and the NDR region often narrowed to $<1 \mathrm{~V}$ in width. This instability at low temperature did not affect the current density of the on/off states previously observed at room temperature (Fig. 4). This indicates that conductions in both on and off is dominated by NP-assisted tunneling and not typical conjugated polymer conduction [as further evidenced by the off state in Fig. 1(a) having a higher current than the "clean" $x$ HTPA devices]. Furthermore, a "super" off state was obo AIP license or copyright, see http://apl.aip.org/apl/copyright.jsp 


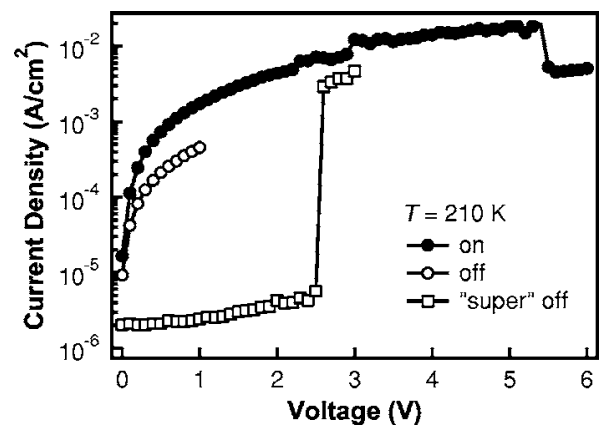

FIG. 4. Typical $I-V$ curves at low temperature (in this plot, $210 \mathrm{~K}$ ). The on and off curves shown here are in the same current density range as is observed at room temperatures. However, the super off state appears (sporadically) only at suppressed temperature. The labels, on, off, and super off indicate the state of the device at the beginning of the (positive) voltage sweep.

served which exhibits a current expected for a clean semiconducting polymer at cryogenic temperature. ${ }^{17}$

The above data suggest a possible mechanism. In the voltage region below $V_{\text {th }}$, the conductivity of the device is determined by the amount of charge trapped on the NPs. These relatively immobilized charges create a space-charge region which reduces the injection barrier, thus increasing the device conductivity. In contrast to the usual effect of space charge on current, this increase in conductivity is due to the build up of charge of opposite sign to the injected charge. At higher bias, some of the trapped charge is either swept away by the field, or eliminated by recombination, leading to NDR.

Analysis of the frequency-dependent capacitance of multiple on and off states suggests the high current to be related to an increase in space charge due to trapped charge on the
NPs. These data also imply that trap sites in the polymer are critical to optimization of device performance and lead to the possibility of decreasing the off state current by using purified or highly ordered polymers.

The authors wish to thank J. Campbell Scott, Luisa Bozano, and Glenn Alers for valuable assistance. This research was funded by a grant from the National Science Foundation (Electrical and Communication Systems Grant No. 0101794).

${ }^{1}$ N. C. C. Lu, IEEE Circuits Devices Mag. 5, 27 (1989).

${ }^{2}$ B. Eitan, P. Pavan, I. Bloom, E. Aloni, A. Frommer, and D. Finzi, IEEE Electron Device Lett. 21, 543 (2000).

${ }^{3}$ J. De Blauwe, IEEE Trans. Nanotechnol. 1, 72 (2002).

${ }^{4}$ J. Akerman, Science 308, 508 (2005).

${ }^{5}$ A. Sheikholeslami and P. G. Gulak, Proc. IEEE 88, 667 (2000).

${ }^{6}$ S. Hudgens and B. Johnson, MRS Bull. 29, 829 (2004).

${ }^{7}$ L. P. Ma, J. Liu, and Y. Yang, Appl. Phys. Lett. 80, 2997 (2002).

${ }^{8}$ J. Ouyang, C.-W. Chu, C. R. Szmanda, L. Ma, and Y. Yang, Nat. Mater. 3, 918 (2004)

${ }^{9}$ L. D. Bozano, B. W. Kean, V. R. Deline, J. R. Salem, and J. C. Scott, Appl. Phys. Lett. 84, 607 (2004).

${ }^{10}$ M. Lauters, B. McCarthy, D. Sarid, and G. E. Jabbour, Appl. Phys. Lett. 87, 231105 (2005).

${ }^{11}$ M. Cölle, M. Büchel, and D. M. de Leeuw, Org. Electron. (in press).

${ }^{12}$ L. D. Bozano, B. W. Kean, M. Beinhoff, K. R. Carter, P. M. Rice, and J. C. Scott, Adv. Funct. Mater. 15, 1933 (2005).

${ }^{13}$ E. H. Nicollian and J. R. Brews, MOS (Metal Oxide Semiconductor) Physics and Technology (Wiley-Interscience, New York, 1982), pp. 176-234.

${ }^{14}$ H. C. F. Martens, H. B. Brom, and P. W. M. Blom, Phys. Rev. B 60, R8489 (1999).

${ }^{15}$ S. Berleb and W. Brütting, Phys. Rev. Lett. 89, 286601 (2002).

${ }^{16}$ H. H. P. Gommans, M. Kemerink, G. G. Andersson, and R. M. T. Pijper, Phys. Rev. B 69, 155216 (2004).

${ }^{17}$ L. Bozano, S. E. Tuttle, S. A. Carter, and P. J. Brock, Appl. Phys. Lett. 73, 3911 (1998). 